\title{
Hypobaric versus isobaric bupivacaine to prevent anesthesia- induced hypotension in elderly patients undergoing surgical repair of hip fracture under continuous spinal anesthesia: a prospective randomized controlled study
}

\author{
Bargaoui A., Raddaoui K., Oueslati M., Bousselmi R., Shili A., Kaabachi O. \\ Mohamed Kassab Institute of Orthopedics.
}

\begin{abstract}
Backgournd and aim of the study :
Anesthesia for hip fracture surgery in the elderly remains controversial. There is no evidence supporting superiority of general or spinal anesthesia [1]. New data suggest that per-operative hypotension may be correlated with mortality [2]. Continuous spinal anesthesia (CSA) preserves hemodynamics better than single shot SA and general anesthesia. However, hypotension is still observed with CSA. Our hypothesis is that unilateral CSA achieved by hypobaric bupivacaine when patients are in the lateral position with the operated side up improves hemodynamic stability.
\end{abstract}

Materials and methods :

It was a prospective randomized controlled study conducted in the Department of anesthesiology and intensive care of Med Kassab Institute of Orthopedics. Local ethics board approved the study and it was registred in the Panafrican Clinical Trials Registry (Id PACTR201606001657480). Patients aged more than 65 years undergoing a surgical repair of a hip fracture were eligible. Patients with any contraindication to spinal aneshesia (severe obstructive valvulopathy, hemostasis impairment, local infection), or patients with predicted difficult assessment (dementia, deafness, etc.) were not included. In the operating room, patients had standard monitoring. Ultrasound guided femoral nerve block was performed to provide analgesia for positioning, Patients were put in the lateral position with the fractured side up. A spinal catheter was introduced via the L4-L5 or L3-L4 interspace. Patients were randomized to 2 groups. Group H received hypobaric bupivacaine, prepared diluting each $\mathrm{ml}$ of $0,5 \%$ isobaric bupivacaine with $1 \mathrm{ml}$ of sterile water. Group I received $0,5 \%$ isobaric bupivacaine. An initial bolus of $2,5 \mathrm{mg}$ was administred. Additional boluses of $2,5 \mathrm{mg}$ were added if necessary until sensitive blockade reached T12 in the fractured side. A slow manual injection with a rate of $1 \mathrm{ml} / 30 \mathrm{sec}$ was used.

Our primary outcomes were the incidence of hypotension (ie, decrease by $20 \%$ of systolic blood pressure) and severe hypotension (decrease by $30 \%$ of systolic blood pressure). Bupivacaine and ephedrine consumption were secondary outcomes. Based on [3], with our hypothesis that hypobaric bupivacaine will reduce the occurrence of hypotension from $30 \%$ to $10 \%$, the sample size for a superiority trial yielded a sample size of 55 patients for each group, with a study power of $80 \%$ and a significance level of 0.05 . Quantitative data were expressed as mean $\pm \mathrm{SD}$ (distibution considered normal as group size $>30)$ Qualitative data were expressed as n (\%). Qualitative data were analyzed by $\chi 2$ test and quantitative data by Student t-test. $\mathrm{P}<0.05$ was considered statistically significant.

Main results and discussion :

One hundred and ten patients were analyzed. The two groups were comparable in age, BMI and ASA class. There was no difference in type and course of surgery. Significantly less patients experienced hypotension and severe hypotension in HB group than in IB group (respectively $53 \%$ vs $73 \%$; $<00,05$ and $53 \%$ vs $22 \%$; $<<0,01$ ). Significantly less ephedrine was used in HB group $(5,6$ vs $1,9 \mathrm{mg}$; $\mathrm{p}<0,01$, difference $\mathrm{CI}$ ). Total consumption of bupivacaine was not significantly different..

Unilateral sensitive blockade was achieved in $37 \%$ of hypobaric group patients. Achieving unilateral blockade may be challenging when the injection is performed via a spinal catheter that may be randomly

\section{References}

[1] Anesthesiology 2012; 117:72-92

[2] Anaesthesia 2016, 71, 506-514

[3] Anesth Analg. 1996 Jun;82(6):1188-91.

[4] Reg Anesth Pain Med. 2001 Sep-Oct;26(5):420-7[

5] Anesth Analg 2006;102:1559-63.

[6] Sao Paulo Med J. 2009; 127(1):7-11.
Table 1. Demographic and surgical data

\begin{tabular}{lcc}
\hline & $\begin{array}{c}\text { H group } \\
(\mathrm{n}=54)\end{array}$ & $\begin{array}{c}\text { I group } \\
(\mathrm{n}=56)\end{array}$ \\
\hline Gender $(\mathrm{M} / \mathrm{F})$ & $23 / 31$ & $21 / 35$ \\
Age $($ years $)$ & $76 \pm 7$ & $78 \pm 6$ \\
Height $(\mathrm{cm})$ & $164 \pm 6$ & $163 \pm 8$ \\
Weight $(\mathrm{kg})$ & $71 \pm 10$ & $67 \pm 12$ \\
BMI $\left(\mathrm{kg} / \mathrm{m}^{2}\right)$ & $26 \pm 3$ & $25 \pm 3$ \\
ASA class (I/I/III) & $13 / 31 / 10$ & $13 / 34 / 9$ \\
Type of surgery & & \\
DHS & 32 & 31 \\
Gamma nail & 12 & 12 \\
Hemiarthroplasty & 10 & 13 \\
Time to operate (days) & $4,9 \pm 1,6$ & $4,4 \pm 2$ \\
Surgical duration (mn) & $68 \pm 19$ & $69 \pm 25$ \\
Hypertension & $28(51 \%)$ & $27(48 \%)$ \\
Coronaropathy & $5(9 \%)$ & $8(14 \%)$ \\
Chronic heart failure & $2(4 \%)$ & $1(2 \%)$ \\
\hline $\begin{array}{l}\text { Data are expressed as ratios, count (percentage) or mean } \pm \text { SD. } \\
\text { There was no significant }\end{array}$ & difference between groups. \\
H=hypobaric bupivacaine; & I=isobaric bupivacaine; \\
DHS=dynamic hip screw. & \multicolumn{3}{l}{}
\end{tabular}

Table 2. Main results

\begin{tabular}{llll}
\hline & $\begin{array}{l}\text { H group } \\
(\mathrm{n}=54)\end{array}$ & $\begin{array}{l}\text { I group } \\
(\mathrm{n}=56)\end{array}$ & $\mathrm{p}$ \\
\hline Unilateral blockade & $20(37 \%)$ & $2(3 \%)$ & $<0,05$ \\
Hypotension & $29(53 \%)$ & $41(73 \%)$ & $<0,05$ \\
Severe hypotension & $30(53 \%)$ & $12(22 \%)$ & $<0,01$ \\
Ephedrine (mg) & $1,9 \pm 4$ & $5,6 \pm 8$ & $<0,01$ \\
Minimal PAS (mmHg) & $109 \pm 18$ & $103 \pm 22$ & $\mathrm{NS}$ \\
$\Delta$ PAS & $22 \pm 11$ & $27 \pm 13$ & $\mathrm{NS}$ \\
Total bupivacaine (mg) & $4,6 \pm 1,8$ & $4,8 \pm 1,8$ & $\mathrm{NS}$ \\
Fluids (ml) & $1247 \pm 464$ & $1282 \pm 327$ & $\mathrm{NS}$ \\
CV complications & $3(5 \%)$ & $3(5 \%)$ & $\mathrm{NS}$ \\
\hline
\end{tabular}

Data are expressed as count (percentage) or mean $\pm \mathrm{SD}$. Blockade is considered unilateral when there are more than 4 metamers difference in sensitive block between operated and non operated sides. $\triangle \mathrm{PAS}=(\mathrm{PAST} 0-$ Minimal PAS $)$ PAST0. $\mathrm{CV}=$ cardiovascular complications (elevated troponin or electrocardiogram change)

directed in the subarachnoid space. Nevertheless, this percentage is consistent with other data [3] and could have been enhanced if we used a slower injection rate [4].

We chose a $20 \%$ and $30 \%$ decrease of the initial systolic blood pressure wich are the most widely used definitions of hypotension and severe hypotension. In our study, we found a higher rate of hypotension than in other studies concerning CSA $[5,6]$. This may be due to prolongedtime to operate on our patients - due to schedule problems or to our management of fluid resuscitation, since we did not use a systematic fluid loading after administering bupivacaine.

One important limitation of our study was the absence of blinding of care provider and data collector, due to different volumes of the anesthetic solutions. One solution could have been to use $0,25 \%$ isobaric bupivacaine in the I group. We did not opt for this solution because we aimed to compare our routine standard of care (CSA with $0,5 \%$ isobaric bupivacaine) with this proposed unilateral CSA with $0,25 \%$ hypobaric bupivacaine. Unilateral

In conclusion, CSA performed with hypobaric bupivacaine in elderly patients undergoing hip fracture surgery in the lateral position with the operated side up may contribute to hemodynamic stability and thus improve mortality. 